\title{
Explaining Rarity of the Dry Grassland Perennial Astragalus exscapus
}

\author{
Thomas Becker
}

(C) The Author(s) 2010. This article is published with open access at Springerlink.com

\begin{abstract}
In Central Europe several plant species of dry grasslands are particularly rare. Here I investigate whether habitat requirements, reproduction, and dispersal potential can contribute to the rarity of Astragalus exscapus (Fabaceae) growing in dry grassland habitats in dry regions of Europe. In addition, I question whether historic events might have contributed to the present-day rarity of $A$. exscapus. To assess habitat requirements of $A$. exscapus, vegetation composition and soil characteristics were studied in 37 populations in central Germany. Production and dispersal potential of seeds were investigated in 10 populations, and germination and recruitment were assessed in experimental plots in three populations. Vegetation of the habitats included most dry grassland community types occurring in the central German dry region indicating a broad ecological niche of the species within dry subcontinental grasslands. Soil characteristics of the habitats also spanned a wide range. Seed production was moderate. $98 \%$ of the seeds sown in the laboratory germinated whereas under natural conditions $20 \%$ of the seeds developed seedlings. Half of these seedlings survived for one year but only $4.5 \%$ for two years. $90 \%$ of the seeds were dispersed less than $50 \mathrm{~cm}$ distance indicating a low dispersal potential. I conclude that $A$. exscapus is mainly limited in dispersal but recruitment limitation might also be important in explaining its rarity. Furthermore, former climate change and postglacial reforestation of the area very likely contribute to the rarity of $A$. exscapus.
\end{abstract}

Keywords Dispersal limitation · Population history $\cdot$ Rare plant $\cdot$ Recruitment limitation $\cdot$ Reproduction $\cdot$ Relict species

Plant nomenclature Wisskirchen and Haeupler (1998) - vascular plants; Koperski et al. (2000) - bryophytes; Scholz (2000) - lichens

\footnotetext{
T. Becker

Department of Biology/Plant Ecology, University of Marburg, Karl-von-Frisch Str. 8, 35043 Marburg, Germany

T. Becker $(\bowtie)$

Department Vegetation and Phytodiversity Analysis, Albrecht von Haller Institute for Plant Science, University of Göttingen, Untere Karspüle 2, 37073 Göttingen, Germany

e-mail: thomas.becker@biologie.uni-goettingen.de
} 


\section{Introduction}

Understanding the ecology of rare plant species is crucial both for understanding causes of their rarity and formulating appropriate management measures. Nonetheless, insufficient understanding of particular species ecology is repeatedly cited as a shortcoming in management of rare and endangered plants (Schemske et al. 1994; Tear et al. 1995). Rare plant species are often characterized by particularly narrow habitat requirements, i.e., they are often associated with habitat types that occur scarcely and are of small geographical area (Kruckeberg and Rabinowitz 1985; Münzbergová and Herben 2005) though not all habitats suitable for a species are necessarily occupied. Identification of suitable but unoccupied habitats may be very important to facilitate beneficial metapopulation dynamics or to select sites for introduction measures for rare species (Maunder 1992; Falk et al. 1996). The most direct way of testing habitat-suitability for a species is to introduce it to a new habitat and test its performance, a method, which is time consuming and often expensive (Wilkinson 2001). An alternative approach is to use information of the accompanying vegetation. This method is based on the common observation that occurrences of species are correlated (Münzbergová and Herben 2004; Ozinga et al. 2005). Here I used relevés and phytosociological units in combination with habitat mapping data to quantify the number and area of unoccupied habitats potentially suitable for the study species. In addition, I measured soil and site characteristics of occupied sites to further assess suitability of unoccupied sites.

Rare plant species may be limited in abundance not only due to habitat specificity, but also due to biological attributes, e.g., low fecundity and low dispersal potential (Bevill and Louda 1999; Giblin and Hamilton 1999; Kaye 1999; Krahulec et al. 1999; Murray et al. 2002; Tremlová and Münzbergová 2007). Several comparisons of rare-common species pairs have demonstrated that reproduction and recruitment are often particularly low in rare plants (Münzbergová 2005; Young et al. 2007). Here I investigate whether seed production, dispersal, germination, seedling emergence, seedling recruitment, and survival of juveniles may contribute to rarity of the study species.

Finally, historic events can determine species' rarity. Historic events including bio-geographical, ecological and anthropogenic factors and the interactions between them over time are rather complex but are presumably often strong determinants for species' rarity (Thompson 2005). Nevertheless, historic events are seldom addressed in questions of plant rarity probably because they are poorly understood (but see Chýlová and Münzbergová 2008). In Central Europe, the turning-point between the Pleistocene and Holocene caused dramatic changes for plant species. Open conifer forests replaced steppe and tundra 10,000 years ago, which were in turn replaced by dense deciduous forests (Walter and Straka 1970). However, on south-facing slopes within dry regions dense forests probably could not develop and there isolated populations of steppe plants were likely able to survive periods when surrounding habitats developed forest cover (Svenning 2002). In the following Neolithic period woodlands were increasingly cleared in many dry regions of Central Europe, artificially recreating grasslands with steppelike conditions and thereby enhancing steppe plants (Pokorný 2005). However, 
many of these new habitats were only colonized by a limited number of steppe plants despite what can be assumed to be sufficient time for colonization. Because relict steppe plants are both legacies of the past and a considerable part of the current central European flora (about 100 species), and because many relict steppe plants are endangered, a better understanding of the causes of rarity in these species is needed. Here I provide ideas on possible effects of historic events on Astragalus exscapus (Fabaceae) considered a relict steppe plant in Central Europe (Walter and Straka 1970). Moreover, this species is not clonal and is bumblebee pollinated as are many other rare plant species in general (Bevill and Louda 1999).

Specifically I ask $i$ ) which habitats are colonized by A. exscapus in Germany, and whether $i i$ ) habitat requirements, iii) characteristics of reproduction, and $i v$ ) dispersal potential of the species contribute to its rarity. Moreover, I ask $v$ ) how rarity of $A$. exscapus may be affected by historic events.

\section{Methods}

\section{Study Species}

Astragalus exscapus L. (Fabaceae; Fig. 1) is a rare, herbaceous, long-lived perennial (at least up to 21 years indicated by root anatomic studies; T. Becker, unpubl. data). This species fits within a rarity type characterized by having small populations of relatively high habitat specificity (Rabinowitz 1981). It has a disjunct distribution in Europe where it is restricted to dry regions (Fig. 2a). In Germany the species occurs on south-facing slopes along river valleys and fault escarpments (Fig. 1). Plants start flowering in April with lemon yellow odoriferous flowers. In dry grasslands on south-facing slopes with high irradiation and a pronounced summer drought period, plants enter into full dormancy in June. In contrast, plants in semidry grasslands retain their leaves until autumn (T. Becker, pers. observation) due probably to a greater soil water availability caused by less intensive evaporation. Pods of $A$. exscapus contain between 1 and 18 bean-shaped seeds (mean \pm s.d.= $3.6 \pm 1.95, n=4220$ pods; T. Becker, unpubl. data). The seeds weigh $2.7-11.7 \mathrm{mg}$ (mean \pm s.d. $=6.8 \pm 1.27 \mathrm{mg}, n=452$ seeds; T. Becker, unpubl. data) and have a smooth surface lacking any special structure to aid dispersal. A. exscapus does not reproduce vegetatively ( $\mathrm{T}$. Becker, pers. observation), which is typical for most Astragalus species (Kaye 1999). The species is self-compatible but is obligately pollinated by insects (bumblebees). Without pollinators the flowers do not produce seeds (Becker et al. 2010). Though insect damage (herbivory) is rare in $A$. exscapus, the plants are often infected by the Astragalus exscapus-specific rust Uromyces jordianus Bubák, which becomes visible when it produces rust-coloured urediospores on the leaves in May. However, it seems that this specialist fungus does not damage the plants significantly. Because A. exscapus is rare and declining it is considered threatened in most countries and throughout its total range (e.g., Korneck et al. 1996; Schnittler and Günther 1999). In Germany, for example, at least 44 populations ( $44 \%$ of all populations known in the country) have become extirpated in the last 150 years (Becker 2003). 


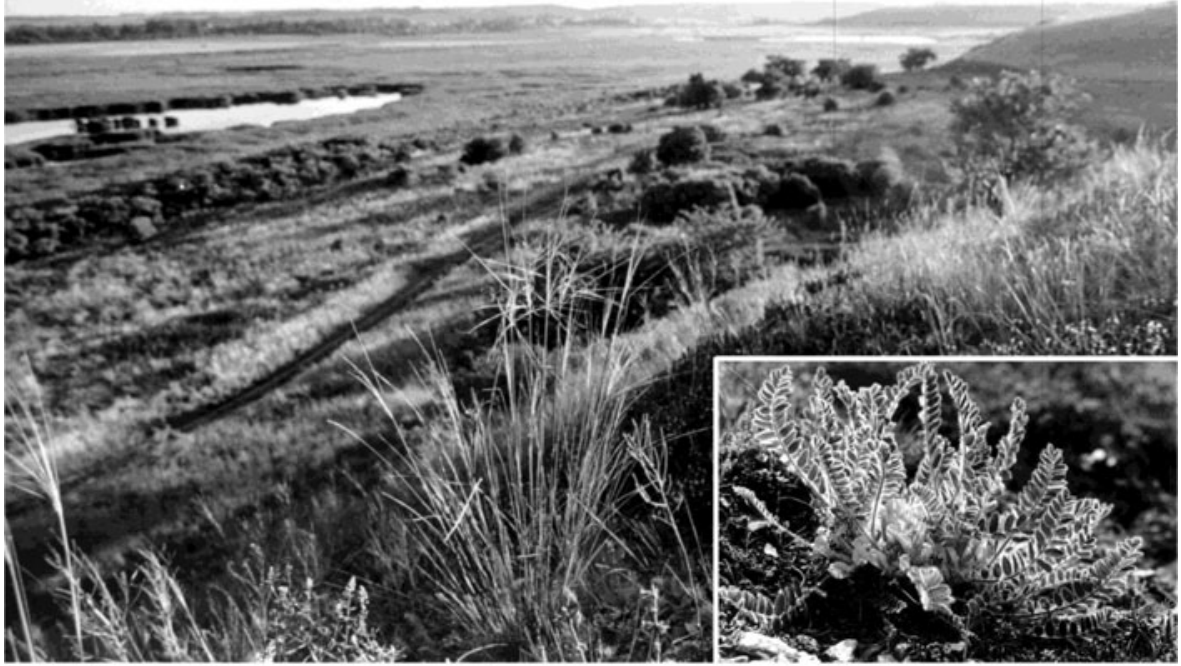

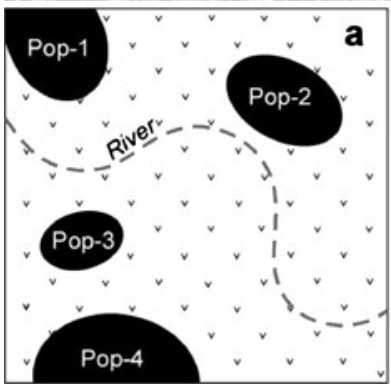

Late Pleistocene period

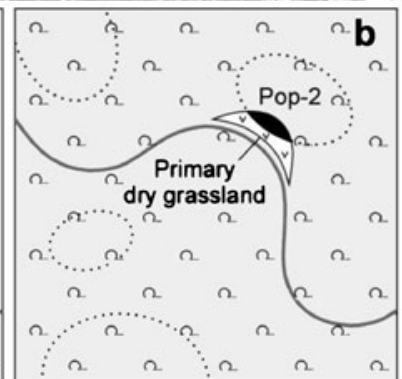

Middle Holocene period

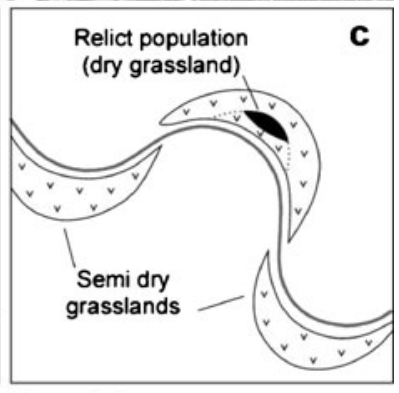

Present day

Fig. 1 Dry grassland habitat of Astragalus exscapus at the margin of the former Lake Salziger See (large picture), and a flowering plant from near Spielberg, Unstrut valley (small picture). The scheme shows a hypothetical scenario of population history of $A$. exscapus (for explanation see text). Dominant vegetation: (a) steppe grasslands, (b) deciduous forests, (c) agricultural fields

\section{Study Region and Study Sites}

This study encompasses the entire range of $A$. exscapus in Germany, i.e., an area extending about $60 \mathrm{~km}$ east-west and $50 \mathrm{~km}$ north-south, located in the central German dry region (Fig. 2b). In total, A. exscapus occurs in 55 populations and a number of distinct sub-populations in Germany. The 37 study populations are located between 75 and 225 ma.s.l. Annual precipitation at the sites ranges from 450 to $500 \mathrm{~mm}$ and mean annual air temperature varies between 8.5 and $9^{\circ} \mathrm{C}$ (Deutscher Wetterdienst 2000). 62\% of the populations are protected by the habitats directive (Natura 2000 areas) and $43 \%$ are additionally located in nature reserves. About two-thirds of the sites are at least sporadically grazed. At the remaining sites succession proceeds slowly because they are dry and of low productivity. Thermophilous oak forest is considered the potential natural 


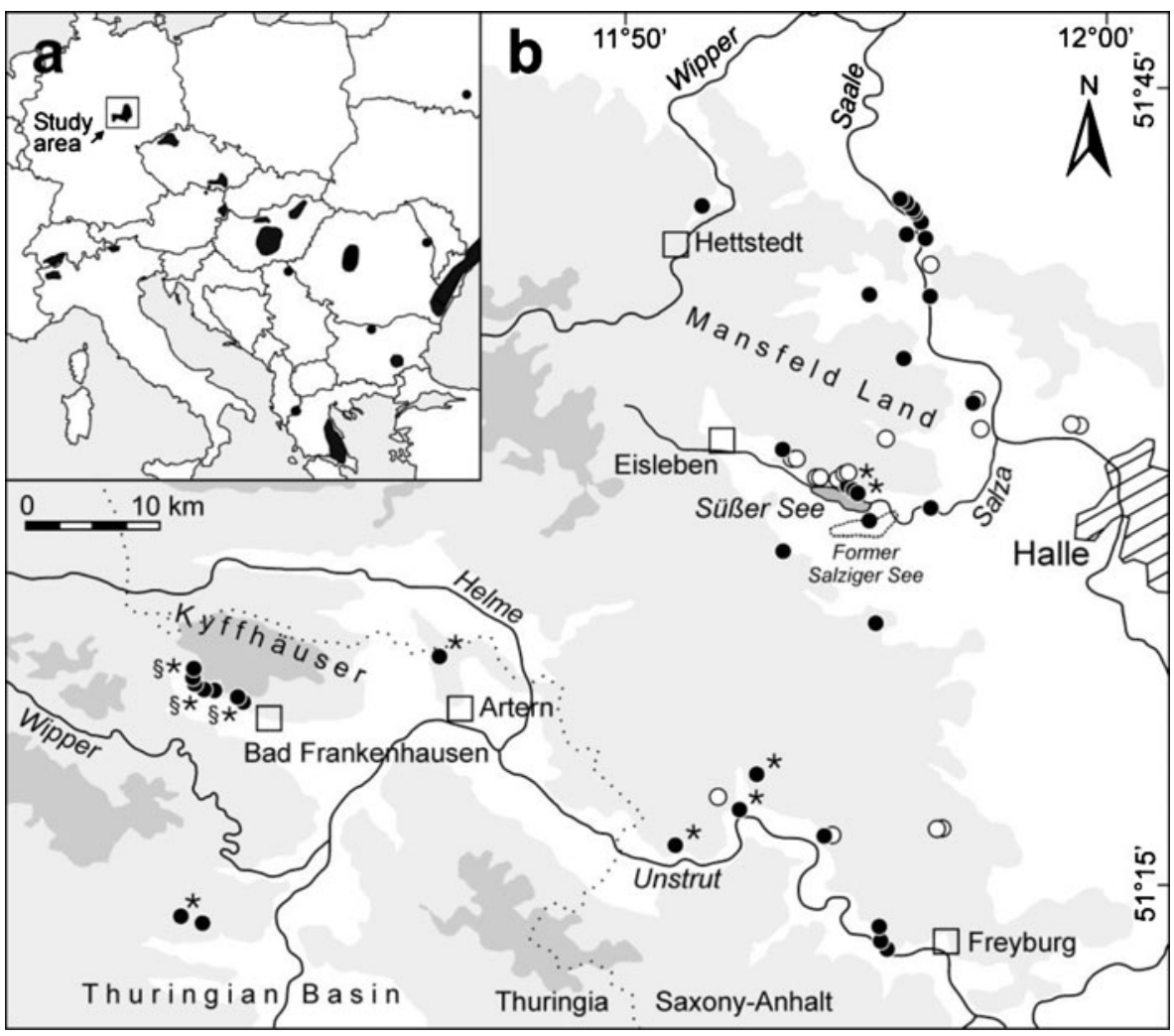

Fig. 2 (a) Entire range, and (b) German sub-range (study region) of Astragalus exscapus. Black dots in b show populations studied and white dots populations not studied. Asterisks $(*)$ indicate populations where dispersal was investigated, and paragraphs $(\S)$ those where germination and recruitment were studied. Map of the total range is modified from Meusel et al. (1965) and other sources. The species was recently reported also from Spain (Ferrández Palacio 2003)

vegetation of the sites (Böhnert et al. 2000). However, at many sites tree growth is limited by drought and therefore small patches of probably natural grasslands occur at these sites. In total, present-day habitats of $A$. exscapus in Germany are distinct islands within a matrix of agricultural land.

\section{Record of Habitat Characteristics}

In 1999 and 2000, species composition and vegetation structure in each of the 37 populations were estimated within three to five plots (in total 170) $2 \times 2$ metres in size using the Braun-Blanquet method. Vegetation plots were distributed regularly within populations and selected on the basis of presence of $A$. exscapus. Number of flowering adults and non-flowering juveniles was counted per plot. Soil depth was measured at five points in each plot by pushing an $85-\mathrm{cm}$-long iron rod into the ground down to bedrock. In each plot five cores were collected from the uppermost soil layer and mixed. Soil pH was measured in a suspension of soil in deionized water and carbonate content was determined using a Scheibler calcimeter. Mean 
unweighted Ellenberg indicator value for moisture was calculated per plot based on vegetation composition (Ellenberg et al. 2001). Index of irradiation was used as a further measure of drought and calculated per plot by equation three following McCune and Keon (2002).

\section{Measuring Reproduction, Seedling Emergence and Dispersal Potential}

Reproduction was estimated using number of flowers, pods, and seeds, seed germination, and recruitment of seedlings. Number of pods was counted among 10 mother plants in each of 10 populations in 1999, and seed production was calculated per mother plant as the number of pods multiplied by mean number of seeds per pod derived from seed counts of 10 pods of each 10 randomly selected (other) plants of the same population. This was done to facilitate investigation of seedling emergence and dispersal potential after natural dissemination. Mother plants were randomly selected on the criterion of 3-m minimum distance to the next fruiting plant to ensure correct assignment of seedlings to mother plants; position of mother plants was noted. Because seedlings were highly accumulated around mother plants and this same pattern was also observed in plants that were even more $(>10 \mathrm{~m})$ isolated from others, correct assignment of seedlings to mother plants is very likely. Mother plants were revisited in April 2000 and the number of naturally emerged seedlings was counted per plant within a radius of one meter. Based on seed production in 1999 the proportion of seedling emergence was estimated per mother plant.

Seed germination was studied both in the laboratory and in the field. In each of five study populations 50 seeds were collected in July 1999 and stored at room temperature in the laboratory until September. Seeds were put into Petri dishes and incubated in a climate cabinet with $20^{\circ} \mathrm{C}$ day temperature and $10^{\circ} \mathrm{C}$ night temperature, and $12 \mathrm{~h}$ light. The number of seedlings was counted every two to three days over three months. In the field, seed germination was studied in three populations in the Kyffhäuser Mountains (Fig. 2). In each population five pairs of $50 \times 50 \mathrm{~cm}$ permanent plots were established in October 2000 at a minimum distance of $3 \mathrm{~m}$ to other plants. In total, 4,480 seeds were collected from the three populations: $1,150,1,380$, and 1,950 seeds, respectively. These came from 15-20 plants per population. In July 2000, 115, 138 and 195 seeds, respectively (each from the same population), were sown in each plot. One plot of each pair was raked after seeding to imitate disturbance by sheep trampling, which is a normal environmental factor for $A$. exscapus because the grasslands were traditionally grazed. Seedlings were counted monthly until August 2001 by N. Voss (Voss 2002) and then once per year in March until 2003. Survival of seedlings was monitored during the same time period.

Seed dispersal potential of $A$. exscapus was measured among the same 100 mother plants out of 10 populations, wherein seedling emergence was estimated as distance of seedlings from their mother plants. Additionally, dispersal direction was measured in relation to slope with a compass considering downhill as the $180^{\circ}$ reading. Wind dispersal potential of the seeds was estimated taking terminal velocity and seed weight into account according to Tackenberg et al. (2003) by dropping 20 seeds from $2 \mathrm{~m}$ height and clocking the time of each drop. 


\section{Data Analysis}

Vegetation was classified using the software COCKTAIL (Bruelheide 2000) based on $u$-value of species' fidelity; only species with $u>3$ were used for classification. For Brachypodium pinnatum, Festuca valesiaca, Sesleria albicans and Stipa capillata, cover was used as an additional factor in classification. In dry continental grasslands these species are important ecologically, and all are useful in syntaxonomy, strongly indicating certain environmental conditions by their abundance. Vegetation types were assigned to syntaxa according Rennwald (2000) and Becker and Brändel (2007). A detrended correspondence analysis (DCA) with $\log (x+1)$-transformed percentage cover values (middle values of the corresponding Braun-Blanquet intervals) and down-weighting of rare species was carried out using the program CANOCO 4.5 (ter Braak and Šmilauer 2002). Univariate ANOVA was used to assess differences in the density of individuals of $A$. exscapus between dry grasslands compared to semi-dry grasslands. Values of environmental characteristics were aggregated on the community level within populations and vegetation cover values and environmental variables that were not normally distributed were $\log (\mathrm{x}+1)$-transformed before statistical calculation to obtain normal distributions. Residuals were checked for normality of distribution. Repeated measurement ANOVA was used to test for the effects of populations, disturbance, and the interaction between population and disturbance on the number of seedlings in experimental plots. Population was used as a random factor and disturbance was used as a fixed factor. The effect of disturbance and the interaction of time (i.e., duration of the experiment) and disturbance were tested against their interaction with population. Dispersal distances of seedlings were modelled by three parameter Lorentzian non-linear curve estimation $y=31.7 /\left(1+((x-43.5) / 43.8)^{2}\right)$ that revealed the highest adjusted $r^{2}$ compared to other fits. Curve estimation was carried out with Sigma Plot 9.0 (SYSTAT Software Inc. 2004). All other analyses were carried out using SPSS 15.0 (SPSS Inc. 2005).

\section{Results}

\section{Habitat Characteristics and Population Density}

Vegetation colonized by $A$. exscapus was assigned to five associations of the class Festuco-Brometea (Table 1). Twelve populations (33\%) occurred partly or completely in Festucetum pallentis grasslands on extremely shallow soils on steep slopes with high irradiation. Within this association the species was found in three subassociations: one on gypsum soil, one on soil containing loess, and one on clayish sandstone soil. Twenty-six populations (70\%) occurred (partly or completely) in Festuco-Stipetum grasslands on deeper soil often containing loess. Within the Festuco-Stipetum four subassociations with $A$. exscapus were differentiated growing on different types of soils: on rocky soils (five populations), deeper calcareous and gypsum soils (six populations), sandy soils (one population), and clayish soils (14 populations). One population was growing in Teucrio-Seslerietum grasslands and five populations (14\%) in Trinio-Caricetum grasslands on calcareous soil. Finally, 21 populations (57\%) 
Table 1 Dry grassland vegetation colonized by Astragalus exscapus in Germany. Percentage frequencies are shown. Brachypodium pinnatum, Festuca valesiaca, Sesleria albicans, and Stipa capillata are listed twice derived by dividing high and low cover values. Only diagnostic species and species with $\geq 25 \%$ frequency in at least one column are shown

\begin{tabular}{|c|c|c|c|c|c|c|c|c|c|c|c|c|}
\hline \multirow{2}{*}{$\begin{array}{l}\text { Vegetation alliance } \\
\text { Column nr. }\end{array}$} & \multicolumn{3}{|c|}{$\begin{array}{l}\text { Festucion } \\
\text { pallentis }\end{array}$} & \multicolumn{4}{|c|}{$\begin{array}{l}\text { Festucion } \\
\text { valesiacae }\end{array}$} & \multicolumn{3}{|c|}{$\begin{array}{l}\text { Xerobromion } \\
\text { erecti }\end{array}$} & \multicolumn{2}{|c|}{$\begin{array}{l}\text { Cirsio-Bra- } \\
\text { chypodion }\end{array}$} \\
\hline & 1 & 2 & 3 & 4 & 5 & 6 & 7 & 8 & 9 & 10 & 11 & 12 \\
\hline Nr. of populations & 8 & 1 & 3 & 5 & 13 & 1 & 6 & 1 & 1 & 3 & 5 & 16 \\
\hline Nr. of relevés & 18 & 3 & 6 & 8 & 34 & 5 & 16 & 3 & 7 & 11 & 11 & 48 \\
\hline Astragalus exscapus & 100 & 100 & 100 & 100 & 100 & 100 & 100 & 100 & 100 & 100 & 100 & \\
\hline
\end{tabular}

Group 1

Festuca pallens

Ceratodon purpureus

Pottia lanceolata

Cephaloziella divaricata

Cladonia foliacea

Cladonia furcata subsp. furcata

Cerastium semidecandrum

Cladonia fimbriata

$\begin{array}{cccc}100 & 100 & 100 & 50 \\ 66 & 100 & 100 & 100 \\ 44 & . & 33 & 37 \\ 27 & 100 & 66 & 12 \\ 16 & 100 & 16 & . \\ 27 & 33 & 16 & . \\ 16 & 66 & 83 & 12 \\ 11 & 33 & 33 & 37\end{array}$

$\begin{array}{cc}20 & 43 \\ \cdot & 25 \\ \cdot & \cdot \\ \cdot & 25 \\ \cdot & 31 \\ \cdot & 25 \\ \cdot & \cdot \\ . & 6\end{array}$

$\begin{array}{cccc}100 & 18 & 18 & . \\ 85 & . & . & 6 \\ 42 & . & . & 2 \\ . & . & . & . \\ . & . & 9 & . \\ . & . & . & . \\ . & . & . & . \\ . & . & . & .\end{array}$

Group 2

Erophila verna agg.

Cerastium pumilum

Bryum caespiticium

Holosteum umbellatum

Toninia sedifolia

Erodium cicutarium

Collema spp.

Arenaria serpyllifolia agg.

Centaurea stoebe

Veronica praecox

Tortula ruralis agg.

Cladonia rangiformis

Acinos arvensis

Taraxacum sect. Erythrosperma

Alyssum alyssoides

Thlaspi perfoliatum

Group 3

Festuca valesiaca $>5 \%$ cover

Stipa capillata $>5 \%$ cover

S. capillata and! F. valesiaca $\leq 5 \%$ cover

$\begin{array}{ccccccc}44 & 66 & 100 & 75 & 73 & 40 & 56 \\ 55 & 33 & 66 & 75 & 76 & 100 & 81 \\ 44 & 100 & 50 & 37 & 35 & . & 25 \\ 38 & 100 & 83 & 12 & 29 & 20 & 12 \\ 38 & . & 16 & 50 & 26 & . & . \\ 5 & . & 50 & 37 & 14 & . & 12 \\ 50 & . & 16 & 37 & 23 & . & . \\ 22 & 66 & 66 & 62 & 38 & 100 & 37 \\ 38 & . & 83 & 87 & 29 & 60 & 12 \\ 11 & 66 & 83 & 12 & 38 & 20 & 6 \\ 11 & . & 50 & 37 & 32 & . & 43 \\ 22 & 100 & 33 & 25 & 35 & 40 & 56 \\ 5 & 33 & 33 & 25 & 2 & 20 & 31 \\ 11 & 66 & 16 & 12 & 11 & . & 31 \\ 11 & . & 16 & 12 & 26 & . & 6 \\ . & 66 & . & 25 & 17 & 100 & 37\end{array}$

$\begin{array}{cccc}57 & \cdot & \cdot & 8 \\ 57 & \cdot & 9 & 20 \\ 100 & 18 & \cdot & 4 \\ 57 & \cdot & \cdot & 6 \\ 42 & \cdot & \cdot & \cdot \\ 28 & \cdot & \cdot & 2 \\ 28 & 18 & \cdot & \cdot \\ \cdot & \cdot & \cdot & 2 \\ . & 18 & \cdot & 6 \\ \cdot & \cdot & \cdot & 6 \\ \cdot & 9 & \cdot & 6 \\ . & \cdot & 27 & 10 \\ . & \cdot & 9 & \cdot \\ 14 & \cdot & \cdot & 4 \\ . & \cdot & \cdot & 2 \\ . & . & \cdot & 2\end{array}$

Group 4

Phleum phleoides

Pseudolysimachion spicatum

Inula hirta

Allium vineale

Geranium sanguineum

Luzula campestris

Arabidopsis thaliana

$\begin{array}{ccccccccccccc}. & . & 50 & 25 & 56 & . & 13 & . & . & . & . & . \\ . & . & 17 & 50 & 26 & . & 13 & . & . & . & . & . \\ 22 & 33 & 50 & 88 & 50 & . & 81 & . & 18 & 33 & 18 & 40\end{array}$


Table 1 (continued)

\begin{tabular}{|c|c|c|c|c|c|c|c|c|c|c|c|c|}
\hline \multirow{2}{*}{$\begin{array}{l}\text { Vegetation alliance } \\
\text { Column nr. }\end{array}$} & \multicolumn{3}{|c|}{$\begin{array}{l}\text { Festucion } \\
\text { pallentis }\end{array}$} & \multicolumn{4}{|c|}{$\begin{array}{l}\text { Festucion } \\
\text { valesiacae }\end{array}$} & \multicolumn{3}{|c|}{$\begin{array}{l}\text { Xerobromion } \\
\text { erecti }\end{array}$} & \multicolumn{2}{|c|}{$\begin{array}{l}\text { Cirsio-Bra- } \\
\text { chypodion }\end{array}$} \\
\hline & 1 & 2 & 3 & 4 & 5 & 6 & 7 & 8 & 9 & 10 & 11 & 12 \\
\hline Nr. of populations & 8 & 1 & 3 & 5 & 13 & 1 & 6 & 1 & 1 & 3 & 5 & 16 \\
\hline Nr. of relevés & 18 & 3 & 6 & 8 & 34 & 5 & 16 & 3 & 7 & 11 & 11 & 48 \\
\hline
\end{tabular}

Scorzonera purpurea

Group 5

Helianthemum nummularium subsp. obscurum

Pleurochaete squarrosa

Stipa pennata

Aster linosyris

Stipa pulcherrima

Onobrychis arenaria

Fumana procumbens

Group 6

Carex humilis

Teucrium montanum

Teucrium chamaedrys

Hippocrepis comosa

Scabiosa canescens

Group 7

Helianthemum canum

Bupleurum falcatum

Group 8

Sesleria albicans $>25 \%$ cover

Onobrychis viciifolia

Group 9

Psora saviczii

Fulgensia bracteata

Ulmus minor

Group 10

Tortella tortuosa

Prunella grandiflora

Bothriochloa ischaemum

Origanum vulgare

Group 11

Briza media

Sesleria albicans $\leq 25 \%$ cover

Pimpinella saxifraga

Viola rupestris

Viola hirta

Potentilla heptaphylla

Group 12

Brachypodium pinnatum $>5 \%$ cover
33

60

33
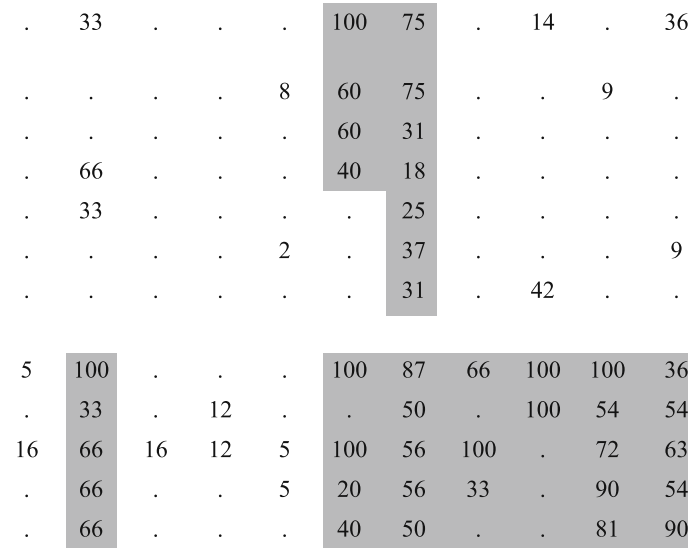

$\begin{array}{ccccccc}100 & 87 & 66 & 100 & 100 & 36 & . \\ . & 50 & . & 100 & 54 & 54 & . \\ 100 & 56 & 100 & . & 72 & 63 & 2 \\ 20 & 56 & 33 & . & 90 & 54 & 6 \\ 40 & 50 & . & . & 81 & 90 & 14\end{array}$

22

\begin{tabular}{ccc|cc}
100 & 100 & 100 & $\cdot$ &. \\
100 & $\cdot$ & 63 & 9 & 10
\end{tabular}

100

100 
Table 1 (continued)

\begin{tabular}{|c|c|c|c|c|c|c|c|c|c|c|c|c|}
\hline \multirow{2}{*}{$\begin{array}{l}\text { Vegetation alliance } \\
\text { Column nr. }\end{array}$} & \multicolumn{3}{|c|}{$\begin{array}{l}\text { Festucion } \\
\text { pallentis }\end{array}$} & \multicolumn{4}{|c|}{$\begin{array}{l}\text { Festucion } \\
\text { valesiacae }\end{array}$} & \multicolumn{3}{|c|}{$\begin{array}{l}\text { Xerobromion } \\
\text { erecti }\end{array}$} & \multicolumn{2}{|c|}{$\begin{array}{l}\text { Cirsio-Bra- } \\
\text { chypodion }\end{array}$} \\
\hline & 1 & 2 & 3 & 4 & 5 & 6 & 7 & 8 & 9 & 10 & 11 & 12 \\
\hline & 8 & 1 & 3 & 5 & 13 & 1 & 6 & 1 & 1 & 3 & 5 & 16 \\
\hline Nr. of relevés & 18 & 3 & 6 & 8 & 34 & 5 & 16 & 3 & 7 & 11 & 11 & 48 \\
\hline Agrimonia eupatoria & . & . & . & . & 14 & . & 6 & . & . & 100 & 36 & 87 \\
\hline Linum catharticum & . & . & . & . & 2 & . & . & 100 & . & 90 & 45 & 56 \\
\hline Medicago lupulina & 5 & . & . & . & 5 & . & . & . & . & 45 & 72 & 68 \\
\hline Campylium chrysophyllum & 5 & . & . & . & 2 & . & 6 & 33 & . & 100 & 36 & 54 \\
\hline Brachythecium rutabulum & . & . & 16 & 12 & 8 & 20 & 19 & 100 & . & 36 & 72 & 64 \\
\hline Cirsium acaule & . & . & . & . & 2 & . & 18 & 33 & . & 54 & 72 & 33 \\
\hline $\begin{array}{l}\text { Lotus corniculatus } \\
\text { subsp. hirsutus }\end{array}$ & 16 & . & . & . & 14 & . & 18 & 33 & . & 72 & 90 & 54 \\
\hline Crataegus monogyna & . & . & . & . & . & 20 & 6 & 33 & . & 27 & 18 & 22 \\
\hline Daucus carota & 5 & . & . & . & . & . & . & . & . & 63 & 27 & 20 \\
\hline Carlina vulgaris & 27 & . & . & . & . & . & . & . & . & 36 & 18 & 37 \\
\hline Plantago media & . & . & . & . & 8 & 60 & 31 & . & . & 63 & 54 & 58 \\
\hline Fissidens dubius & 16 & . & . & . & 11 & 60 & 31 & . & . & 100 & 36 & 54 \\
\hline Fragaria viridis & 5 & . & . & . & 5 & 60 & . & 33 & . & 72 & 45 & 37 \\
\hline Adonis vernalis & . & . & . & . & . & 40 & 18 & . & . & 63 & 27 & 16 \\
\hline Scabiosa ochroleuca & 5 & . & . & 12 & 5 & 60 & 6 & 33 & . & 81 & . & 52 \\
\hline Ranunculus bulbosus & . & . & . & . & 2 & . & 6 & . & . & . & . & 35 \\
\hline \multicolumn{13}{|l|}{ Further vascular plants } \\
\hline Euphorbia cyparissias & 83 & 100 & 83 & 87 & 91 & 80 & 100 & 100 & 85 & 100 & 63 & 81 \\
\hline Thymus praecox & 83 & 66 & 66 & 37 & 38 & 40 & 87 & 100 & 100 & 100 & 100 & 64 \\
\hline Festuca rupicola & 72 & . & 50 & 62 & 73 & 100 & 68 & 100 & . & 100 & 100 & 97 \\
\hline Koeleria macrantha & 66 & . & 100 & 62 & 94 & 100 & 81 & . & 14 & 81 & 54 & 79 \\
\hline Potentilla incana & 66 & 100 & 33 & 87 & 61 & 60 & 100 & . & 42 & 27 & 54 & 72 \\
\hline $\begin{array}{l}\text { Brachypodium pinnatum } \\
\leq 5 \% \text { cover }\end{array}$ & 50 & . & . & 12 & 55 & 100 & 31 & 100 & . & 100 & 100 & 85 \\
\hline Achillea pannonica & 33 & 33 & 50 & 12 & 55 & 100 & 31 & 33 & . & 90 & 18 & 83 \\
\hline Sanguisorba minor & 72 & . & 16 & 62 & 38 & . & 31 & 33 & 42 & 100 & 63 & 56 \\
\hline Salvia pratensis & 16 & . & 33 & 75 & 58 & 80 & 62 & . & . & 72 & 36 & 50 \\
\hline Eryngium campestre & 38 & . & 50 & 37 & 73 & 100 & 56 & . & . & 36 & 18 & 72 \\
\hline Bromus erectus & 38 & . & . & 87 & 26 & . & 56 & . & 100 & 45 & 72 & 35 \\
\hline Galium verum & 5 & . & . & 25 & 35 & 80 & 50 & . & . & 81 & 63 & 68 \\
\hline Falcaria vulgaris & 33 & 33 & 50 & 25 & 38 & 100 & 50 & 33 & . & . & 9 & 18 \\
\hline Rosa rubiginosa & 22 & . & 16 & 12 & 8 & . & 31 & 66 & 14 & 100 & 54 & 29 \\
\hline Hieracium pilosella & 50 & 33 & 33 & . & 26 & . & 12 & . & . & 90 & 18 & 62 \\
\hline Potentilla tabernaemontani & . & . & 50 & . & 20 & 40 & . & 33 & 42 & 81 & 27 & 27 \\
\hline Galium glaucum & 16 & 66 & 83 & 25 & 41 & 40 & 12 & . & . & 9 & . & 27 \\
\hline Helictotrichon pratense & . & . & . & . & 20 & 60 & 62 & . & . & 81 & 45 & 47 \\
\hline Asperula cynanchica & 22 & . & 50 & 37 & 26 & . & 37 & . & . & 72 & 18 & 39 \\
\hline Poa angustifolia & 27 & . & . & . & 47 & 80 & 12 & . & . & 18 & 45 & 70 \\
\hline
\end{tabular}


Table 1 (continued)

\begin{tabular}{|c|c|c|c|c|c|c|c|c|c|c|c|c|}
\hline \multirow{2}{*}{$\begin{array}{l}\text { Vegetation alliance } \\
\text { Column nr. }\end{array}$} & \multicolumn{3}{|c|}{$\begin{array}{l}\text { Festucion } \\
\text { pallentis }\end{array}$} & \multicolumn{4}{|c|}{$\begin{array}{l}\text { Festucion } \\
\text { valesiacae }\end{array}$} & \multicolumn{3}{|c|}{$\begin{array}{l}\text { Xerobromion } \\
\text { erecti }\end{array}$} & \multicolumn{2}{|c|}{$\begin{array}{l}\text { Cirsio-Bra- } \\
\text { chypodion }\end{array}$} \\
\hline & 1 & 2 & 3 & 4 & 5 & 6 & 7 & 8 & 9 & 10 & 11 & 12 \\
\hline Nr. of populations & 8 & 1 & 3 & 5 & 13 & 1 & 6 & 1 & 1 & 3 & 5 & 16 \\
\hline Nr. of relevés & 18 & 3 & 6 & 8 & 34 & 5 & 16 & 3 & 7 & 11 & 11 & 48 \\
\hline Medicago falcata & 5 & 33 & 33 & 25 & 35 & 20 & 50 & . & . & 36 & 27 & 33 \\
\hline Dactylis glomerata & 16 & . & . & 25 & 17 & 80 & 6 & 33 & . & 9 & 36 & 70 \\
\hline Centaurea scabiosa & 5 & . & . & 12 & 2 & 60 & . & 100 & . & 18 & 9 & 18 \\
\hline Stachys recta & 5 & . & 33 & 12 & 11 & 100 & 25 & . & . & 9 & 9 & 6 \\
\hline Dianthus carthusianorum & 5 & 33 & 33 & 25 & 20 & 20 & 18 & . & . & . & 18 & 31 \\
\hline Prunus spinosa & 22 & 33 & . & 37 & . & 40 & 6 & . & . & 9 & 54 & 2 \\
\hline Artemisia campestris & 11 & 66 & 50 & 37 & 17 & . & 12 & . & . & . & . & 2 \\
\hline Ononis spinosa agg. & 22 & . & . & . & 32 & . & 6 & 66 & . & 9 & . & 56 \\
\hline Rosa canina & 5 & . & 16 & 37 & 11 & 20 & 25 & . & 14 & 27 & 18 & 14 \\
\hline Anthericum liliago & 11 & 66 & . & 25 & 2 & 40 & 25 & . & . & 9 & . & 2 \\
\hline Convolvulus arvensis & 38 & . & 16 & 62 & 29 & . & . & . & . & 9 & . & 6 \\
\hline Astragalus danicus & . & 33 & . & . & 2 & . & 37 & . & . & 18 & 54 & 10 \\
\hline Erysimum crepidifolium & 22 & . & 33 & 50 & 32 & . & . & . & . & . & . & 6 \\
\hline Cuscuta epithymum & 11 & . & 33 & 12 & 2 & . & 18 & . & . & 36 & 18 & 2 \\
\hline Lithospermum arvense sibthorpianum & 5 & 66 & 33 & 12 & . & . & 6 & . & . & . & . & . \\
\hline Seseli hippomarathrum & 11 & . & 16 & 37 & 17 & . & . & . & . & 9 & 18 & 2 \\
\hline \multicolumn{13}{|l|}{ Further mosses and lichens } \\
\hline $\begin{array}{l}\text { Hypnum cupressiforme } \\
\text { var. lacunosum }\end{array}$ & 27 & 33 & 66 & 25 & 58 & 40 & 87 & . & . & 36 & 90 & 56 \\
\hline Homalothecium lutescens & 16 & 33 & 16 & 12 & 50 & 40 & 43 & 66 & . & 45 & 45 & 68 \\
\hline Weissia spp. & 55 & . & 50 & 37 & 35 & 40 & 31 & . & 100 & 54 & . & 25 \\
\hline Bryum capillare agg. & 61 & 66 & 50 & 37 & 26 & 20 & 50 & . & 14 & . & 36 & 8 \\
\hline $\begin{array}{l}\text { Cladonia furcata subsp. } \\
\text { subrangiformis }\end{array}$ & 22 & . & 50 & 25 & 23 & . & 43 & . & 14 & 36 & 45 & 12 \\
\hline Didymodon fallax & 61 & 33 & 33 & 37 & 20 & . & 6 & . & 14 & 18 & 9 & 22 \\
\hline Thuidium abietinum & 11 & . & 16 & 25 & 32 & . & 56 & . & . & 63 & 18 & 29 \\
\hline
\end{tabular}

Column nr.

1 - Festucetum pallentis typicum; 2 - F. p. teucrietosum; 3 - F. p. stipetosum; 4 - Festuco-Stipetum festucetosum; 5 - F.S. typicum; $6-$ F.S. phleetosum; 7 - F.-S. teucrietosum; 8 - Teucrio-Seslerietum adonietosum; 9 - Trinio-Caricetum festucetosum; 10 - T.-C. adonietosum; 11 - Adonido-Brachypodietum teucrietosum; 12 - A.-B. typicum.

were growing in Adonido-Brachypodietum grasslands with mesic conditions, i.e., deeper soils on gentle slopes with less irradiation. Within this association A. exscapus occurs in two subassociations on calcareous (five populations) and siliceous soil (16 populations).

Sandstone (21 populations) was the most common substrate of $A$. exscapus, followed by gypsum (10 populations) and limestone (seven populations); these substrate types originated from several geological periods (mostly from the Triassic but also Permian, Carboniferous, and Tertiary) and thus differed in their chemical and physical characteristics also within substrate types. In addition, loess was a common substrate (17 populations) but was often only admixed to the other 
substrates and most of the concerning populations were only partly growing on loess soil.

Slope, soil depth, carbonate content of the soil, cover and height of the vegetation, and proportion of open soil ranged considerably within and among populations while $\mathrm{pH}$ of the soil, southerliness of aspect, incident radiation and indicator values for moisture showed less variation (Table 2). However, the first axis of a DCA ordination (eigenvalue $0.39,10 \%$ of variance explained) was strongly positively correlated to the indicator value for moisture $(r=0.68 ; P<0.001)$ and soil depth $(r=0.33 ; P<0.001)$, and negatively to incident irradiation $(r=-0.40 ; P<0.001)$, indicating that water availability is the main cause of habitat variation in populations of $A$. exscapus.

The density of adult individuals of $A$. exscapus was significantly higher in semi-dry grasslands (Cirsio-Brachypodion) compared to dry grasslands (Festucion pallentis, $F$. valesiacae, Xerobromion) (11.2 vs 6.9 plants per $\left.\mathrm{m}^{2} ; F=4.7 ; P<0.035 ; n=49\right)$. The density of juvenile plants was marginally higher in semi-dry than in dry grasslands (2.5 vs 0.8 plants per $\left.\mathrm{m}^{2} ; F=3.4 ; P<0.070 ; n=47\right)$.

\section{Reproduction and Dispersal Potential}

In 1999 the number of flowers per plant ranged between 1 and 405 (mean \pm s.d. $=127 \pm$ $84, n=100$ plants), and the number of pods between 0 and 183 (mean \pm s.d. $=52 \pm$ $59, n=100$ plants). Seed set of the 100 plants ranged between 3 and 666 seeds. On average, plants produced $227($ s.d. $=247)$ viable seeds. Per plant $0.0 \%$ to $80 \%$ of developed seeds (on average 13.5 (s.d. $=21 \%$ ), $n=100$ plants) were eaten by parasitic wasps (Eurytoma sp.), i.e., these seeds were no longer germinable. Only $1.1 \%$ of the remaining seeds that disseminated naturally in 1999 became seedlings in the following year, i.e., 0 to 34 seedlings (on average 3.2 seedlings, $n=100$

Table 2 Environmental variables of the habitats of Astragalus exscapus in Germany based on 170 plots of 37 populations. Mean values, standard deviations, and ranges are given. Aspect is represented by degrees from northeast to southwest, with a value of $0=45^{\circ}$ (coolest $/$ moistest aspect) and $1=225^{\circ}$ (warmest/driest aspect). Incident radiation index ranges $0.44-1.15$ (lowest to highest irradiation)

\begin{tabular}{lcccc}
\hline & Mean & s.d. & Min. & Max. \\
\hline Slope $\left(^{\circ}\right)$ & 25 & 9.8 & 0 & 47 \\
Southerliness of aspect & 0.83 & 0.13 & 0.44 & 1.00 \\
Incident radiation (index) & 0.99 & 0.10 & 0.65 & 1.14 \\
Soil depth (cm) & 37 & 21 & 3 & $>85$ \\
pH of the soil & 7.4 & 0.4 & 5.0 & 8.2 \\
Carbonate content of the soil (\%) & 12 & 9.6 & 2.4 & 55 \\
Indicator value of moisture & 3.1 & 0.3 & 25 & 4.0 \\
Cover of herb layer (\%) & 75 & 17 & 3 & 100 \\
Height of herb layer (\%) & 14 & 7 & 0 & 35 \\
Cover of moss layer (\%) & 19 & 23 & 1 & 90 \\
Cover of litter layer (\%) & 32 & 27 & 0 & 100 \\
Proportion of open soil (\%) & 12 & 13 & 76 \\
\hline
\end{tabular}


plants) were counted per mother plant. By contrast, $15 \%$ of the seeds sown within experimental plots in 2000 became seedlings in the following year, and a total of $20 \%$ of seeds germinated within the three-year period of investigation (Fig. 3). $90 \%$ of these seeds germinated during the first year and $10 \%$ in the second year. Germination mainly occurred in late winter and early spring. 52\% of these seedlings survived the first year but only $4.5 \%$ continued to live through the second year. Disturbance had a marginal positive effect on emergence of seedlings (Table 3). Additionally, the proportion of emerged seedlings over time was higher in disturbed than in undisturbed plots indicated by a significant interaction between disturbance and time. There was no interaction effect of population $\times$ treatment or of population $\times$ time. The proportion of emerged seedlings per population ranged from $15 \%-26 \%$ and differed marginally among populations.

$70 \%$ of the seedlings disseminated naturally occurred within less than $30-\mathrm{cm}$ distance from their mother plants, and $90 \%$ in less than $50-\mathrm{cm}$ distance (Fig. 4a). Mean distance of seedlings to mother plants was $24 \mathrm{~cm}$. Direction of dispersal was clearly down-hill (Fig. 4b). However, distance and slope were not correlated ( $r=-0.11, P=0.34, n=93$ plants). Mean terminal velocity of the seeds was $0.33 \mathrm{sec}$ per meter indicating very low wind dispersal potential, i.e., wind dispersal potential corresponds to class one on the nine-sectional scale according to Tackenberg et al. (2003). Only two seedlings were found (by chance) in ca. 5-m distance to their mother plant, both in the downhill direction. Incidentally, ants were repeatedly observed carrying seeds.

\section{Discussion}

\section{Do Habitat Requirements Contribute to the Rarity of A. exscapus?}

The assessment of habitat requirements of $A$. exscapus was based on occupied plots, i.e., my study cannot detect for possible differences between occupied and

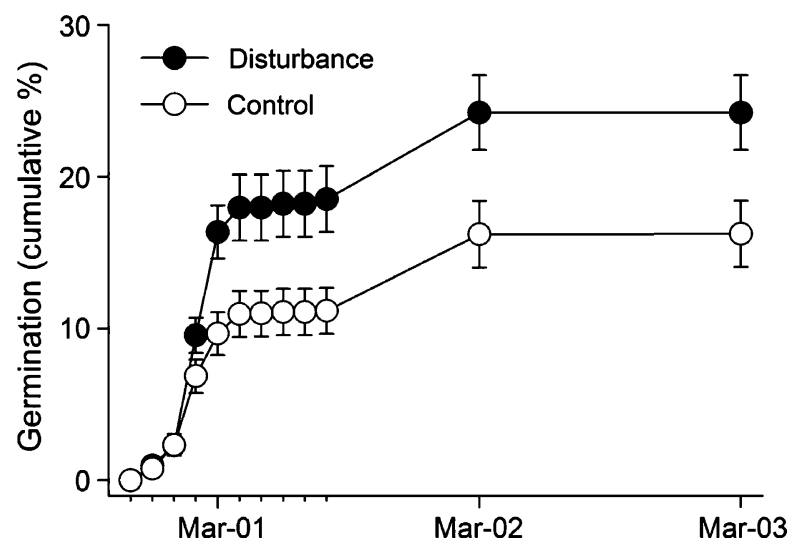

Fig. 3 Cumulative number of seedlings emerged from seeds that were sown on experimental plots within three populations of Astragalus exscapus under two treatments over three years. Mean and 1 s.e. are given. Each of 15 plots per treatment were used as replicates 
Table 3 Effect of population, disturbance, and time on seed germination in Astragalus exscapus. Repeated measurement ANOVA

\begin{tabular}{lcccc}
\hline & d.f. & MS & $F$ & $P$ \\
\hline Population & 2.0 & 990 & 3.3 & 0.052 \\
Disturbance & 1.0 & 2333 & 11.2 & 0.079 \\
Population $\times$ disturbance & 2.0 & 209 & 0.7 & 0.504 \\
Time & 1.4 & 11243 & 28.9 & 0.033 \\
Time $\times$ population & 2.9 & 390 & 6.5 & 0.134 \\
Time $\times$ disturbance & 1.4 & 631 & 10.5 & 0.003 \\
Time $\times$ population $\times$ disturbance & 2.9 & 60 & 0.7 & 0.540 \\
Error & 34.8 & 83 & & \\
\hline
\end{tabular}

unoccupied sites in a direct manner. However, the 12 syntaxa found to be habitat for A. exscapus account for most of the dry grassland sites in the central German dry region and habitat characteristics of the sites spanned a wide range of dry grasslands, i.e., the species was not restricted to a certain substrate, grassland type or vegetation structure as are many other dry grassland species (this result has already been observed within one A. exscapus population by Becker and Voß 2003). Therefore, I presume that within its distributional range in Saxony-Anhalt, the species can grow in most of the 925 dry grassland sites regarded as "steppe grasslands" and "semi-dry grasslands" (Natura 2000-Codes *6211 and *6213) as classified by the European Habitats Directive (92/43/EEC; European Community 1992). In this scenario the 31 sites occupied by the species, which collectively cover an area of only 3.4 hectares, means that $96.6 \%$ of the presumably potential sites and $99.6 \%$ of the potential area of a total
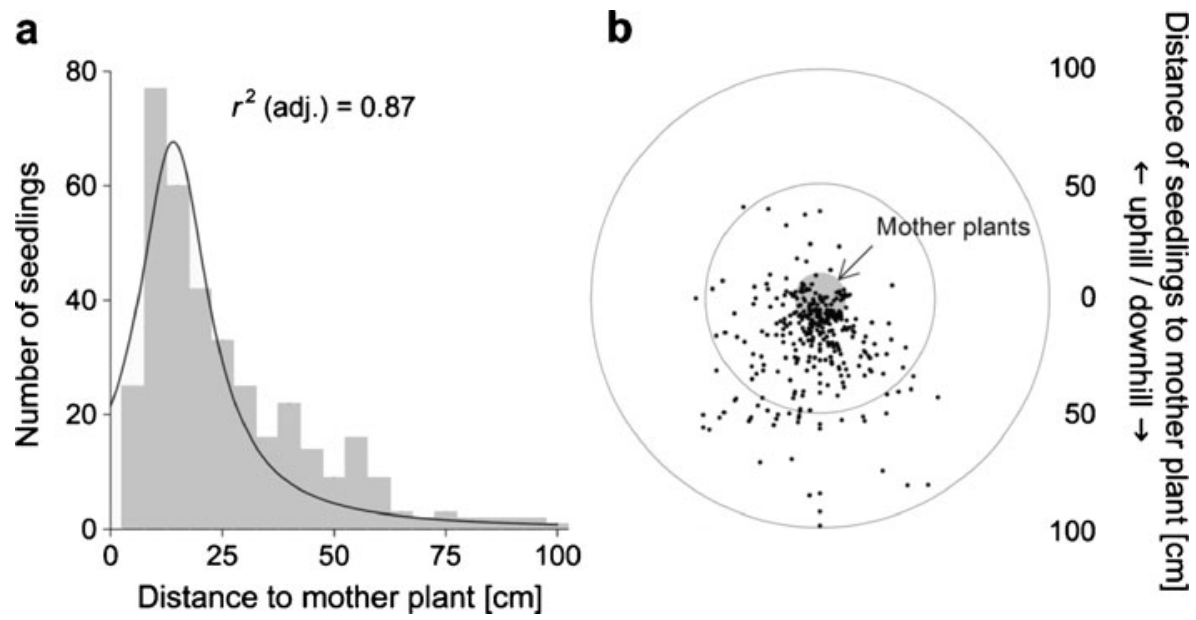

Fig. 4 (a) Distance and (b) direction of dispersal of Astragalus exscapus measured by seedlings. Dispersal distance was modelled by three parameter Lorentzian non-linear curve estimation $y=31.7 /\left(1+((x-43.5) / 43.8)^{2}\right)$. Part b shows the position of 365 seedlings (dots) found in 100 putative mother plants, i.e., 100 mother plants and their seedlings were projected over each other within the chart 
of 894 hectares are not occupied by the species. Of these, only dry grasslands on north-facing slopes (which are rare within the region) might be unsuitable for the species, as evidenced by its being restricted to south-facing slopes. Therefore, in total, the rarity of $A$. exscapus cannot be well explained by habitat limitation.

The positive relationship of $A$. exscapus to sandstone substrate can be explained by the relative abundance of this substrate type within the region, i.e., sandstone is the most abundant surface bedrock type in the region and therefore is colonized by more populations.

Our finding that dry grassland vegetation is characteristic for relevés that include $A$. exscapus indicate that dry grasslands are a more suitable habitat for the species than semi-dry grasslands. However, the positive association between A. exscapus and dry grasslands may be also explained by historic factors, in that dry grasslands more than semi-dry grasslands represent sites where the species would have survived forest expansion in the mid-Holocene (see Fig. 1a,b). Indeed, many of the current populations of $A$. exscapus occur exactly at the driest sites of e.g., former undercut river slopes; many of these sites probably were never completely wooded. In this scenario semi-dry grasslands are either not yet completely colonized (maybe due to the species low dispersal potential) or they are less colonized possibly because they are less stable, i.e., succession proceeds faster than in dry grasslands and may even change over to woodland. Therefore, semi-dry grassland populations of $A$. exscapus might bear a higher risk of extinction. However, there was a higher density of A. exscapus in semi-dry grasslands where in fact most of the large populations occur (Becker 2003). This suggests that semi-dry grasslands are a more suitable habitat for A. exscapus in general though most populations occur in dry grasslands. Thus, as a practical consequence, populations in semi-dry grassland should be considered for effective conservation efforts, e.g., when reintroducing A. exscapus into formerly occupied sites. Reintroduction efforts for $A$. exscapus are currently intended by the Ministry for Agriculture, Conservation and Environment of the Federal State of Thuringia as part of a project of conservation of steppe grasslands supported by the LIFE programme of the European Union. Considering semi-dry grasslands as a habitat for $A$. exscapus might also become important if global warming will cause more extreme conditions in dry grasslands making this habitat less suitable for A. exscapus in the future (Sternberg et al. 1999; Sparks and Menzel 2002).

\section{Do Reproduction and Dispersal Potential Contribute to the Rarity of A. exscapus?}

Astragalus exscapus plants have considerable reproductive potential higher than e.g., in the long-lived Astragalus australis var. olympicus, a rare endemic in the US (Kaye 1999). However, reproductive potential of $A$. exscapus could have been reduced when its habitats were very small and surrounded by extensive forest in the mid-Holocene. The former can be assumed because A. exscapus depends on bumblebees for pollination (without pollinators A. exscapus does not produce seeds; Becker et al. 2010), and bumblebees are known to avoid woodland habitats (Westrich 1989). Therefore, many populations of $A$. exscapus could have gone extinct due to probably low reproduction in that time, contributing to the present day rarity of the species.

Predispersal seed predation in A. exscapus by parasitic wasps was locally intense, damaging up to $80 \%$ of the developed seeds per population. However, the overall 
predation rate of $13.5 \%$ is not high and even lower than in other rare as well as more common Astragalus species (Green and Palmblad 1975; Youtie and Miller 1986; Kaye 1999). In total, present day seed production seems not as a strongly limiting factor for $A$. exscapus. However, the proportion of emerged seedlings was considerably lower in experimental plots than in the laboratory. This may be due to seed predation, rather than to seed dormancy, which is unlikely given that in the experimental plots all seedlings emerged in the first two years. Most legumes have transient, not permanent seed banks (Baskin and Baskin 1998).

The proportion of seedlings emerged was considerably lower among naturally disseminated seeds than in seeds that were sown. This could be due to seed/seedling predation and competition of mother plants. However, competition of mother plants could hardly affect seedlings because adult plants are without leaves at the time of germination and early growth in late winter and early springs, and so light and moisture conditions are suitable for seedlings at that time even in the presence of adult plants. In contrast, seed predation may more convincingly explain the low proportion of seedlings emerged under natural conditions. High rates of seed predation have also been found in other rare plants and contribute to the explanation of the rarity of some species (Münzbergová 2005). In A. exscapus, disseminated seeds lie densely clustered on the soil surface under mother plants and thus may attract seed-predators. I found empty pods with the bite marks of mice in several populations. These were often in the vicinity of woodland edges; a common habitat of mice. The higher proportion of emerged seedlings in raked plots with seeds manually incorporated into the soil can be explained by lower seed predation and better recruitment conditions.

The significant interaction between disturbance and time in the recruitment experiment is mainly caused by a higher proportion of emergent seedlings in disturbed plots in late winter and early spring of the first year. The proportion of emerged seedlings differed marginally among populations and was highest in the Kosakenstein population. This population was characterized by apparently good conditions of emergence: a high proportion of the area having open and loose soil rich in nutrients, as indicated by high abundance and proper growth of many annuals.

In my study seedling mortality (in the first year) was low, while seedling mortality of juveniles (second year) was rather high. Many studies found the opposite pattern, i.e., much higher mortality among seedlings than juveniles (e.g., Maschinski et al. 2004). Because there were no noteworthy extreme climate events during the time of the study, this high juvenile mortality cannot be explained.

The low dispersal potential in A. exscapus concurs with other studies considering a low dispersal potential as a cause of plant species rarity (Kunin and Gaston 1993). Low dispersal potential is also in line with my observation that juvenile plants always occur close to mother plants, resulting in a clustered distribution. It should be noted that $A$. exscapus is listed as a wind-dispersed species (anemochorous) by Jäger and Werner (2005) possibly in reference to the North American Astragalus australis var. olympicus for which wind dispersal of seeds within pods has been observed (Kaye 1999). In A. exscapus, however, dispersal of seeds within pods (and thus wind-dispersal) is extremely unlikely, because the seeds dehisce from the pods more readily than the pods drop from the mother plants ( $T$. Becker, pers. observation). Indeed, the pods split open and the seeds disperse very soon after ripening. 
However, migration over large distances must have somehow occurred when the species once colonized the region probably from Armenia or Kurdistan, the geographical centre of the old-world Astragalus section Caprini DC. (Podlech 1988), and possibly over long distances by seeds on the hooves of ungulates (which were abundant in Pleistocene steppes) or, alternatively, migration might have been gradual in a stepwise fashion over a very long time.

Acknowledgements I kindly thank Nicole Voss for starting the recruitment experiment in the field, the Kyffhäuser Natural Park Administration (Carmen Rosenstock, Jörg Nonnen) for friendly hospitality, Dieter Frank, State Office for Environment Saxony-Anhalt, for providing habitat mapping data, Mark Frenzel, for determination of parasitic wasps, and Volker Kummer for mycological information. Ute Becker, Ulrich Deil, Hartmut Dierschke and three anonymous referees gave valuable comments to earlier drafts of this paper, and Curtis Björk made linguistic corrections. The field research was funded by the German Federal Environmental Foundation ("Deutsche Bundesstiftung Umwelt").

Open Access This article is distributed under the terms of the Creative Commons Attribution Noncommercial License which permits any noncommercial use, distribution, and reproduction in any medium, provided the original author(s) and source are credited.

\section{References}

Baskin CC, Baskin JM (1998) Seeds - ecology, biogeography and evolution of dormancy and germination. Academic Press, San Diego

Becker T (2003) Auswirkungen langzeitiger Fragmentierung auf Populationen am Beispiel der reliktischen Steppenrasenart Astragalus exscapus L. (Fabaceae). Diss Bot 380:1-210

Becker T, Brändel M (2007) Vegetation-environment relationships in a heavy metal-dry grasslandcomplex. Folia Geobot 42:11-28

Becker T, Voß N (2003) Einnischung der seltenen Steppenrasenart Astragalus exscapus L. (Stengelloser Tragant) im Kyffhäusergebirge (Thüringen, Deutschland). Feddes Repert 114:142-165

Becker T, Voss N, Durka W (2010) Pollen limitation and inbreeding depression in an "old rare" bumblebee-pollinated grassland herb. Pl Biol (in press)

Bevill RL, Louda SM (1999) Comparisons of related rare and common species in the study of plant rarity. Conservation Biol 13:493-498

Böhnert W, Federschmidt A, Köck UV, Refior K, Stöcker G, Wartemann G (2000) Karte der Potentiellen Natürlichen Vegetation von Sachsen-Anhalt. Berichte des Landesamtes für Umweltschutz SachsenAnhalt, Sonderheft 1/2000, Landesamt für Umweltschutz Sachsen-Anhalt, Halle

Bruelheide H (2000) A new measure of fidelity and its application to defining species groups. $J$ Veg Sci 11:167-178

Chýlová T, Münzbergová Z (2008) Past land use co-determines the present distribution of dry grassland plant species. Preslia 80:183-198

Deutscher Wetterdienst (2000) Witterungsreport. Offenbach, Main

Ellenberg H, Weber HE, Düll R, Wirth V, Werner W (2001) Zeigerwerte von Pflanzen in Mitteleuropa. Scripta Geobot 18:1-262

European Community (1992) European Community Directive 92/43/EEC on the Conservation of natural habitats and of wild fauna and flora (Habitat Directive). European Community, Brussels

Falk DA, Millar CI, Olwell M (1996) Restoring diversity: Strategies for reintroduction of endangered plants. Island Press, New York

Ferrández Palacio JV (2003) Astragalus exscapus L. (Leguminosae), nueva especie para la flora de la Península Ibérica. Coll Bot 26:119-124

Giblin DE, Hamilton CW (1999) The relationships of reproductive biology to the rarity of endemic Aster curtus (Asteraceae). Canad J Bot 77:140-149

Green TW, Palmblad IG (1975) Effects of insect seed predators on Astragalus cibarius and Astragalus utahensis (Leguminosae). Ecology 56:1435-1440

Jäger EJ, Werner K (eds) (2005) Rothmaler Exkursionsflora von Deutschland, 4. Elsevier, München 
Kaye TN (1999) From flowering to dispersal: reproductive ecology of an endemic plant, Astragalus australis var. olympicus (Fabaceae). Amer J Bot 86:1248-1256

Koperski M, Sauer M, Braun W, Gradstein SR (2000) Referenzliste der Moose Deutschlands. Schriftenreihe Vegetationsk 34:1-519

Korneck D, Schnittler M, Vollmer I (1996) Rote Liste der Farn- und Blütenpflanzen (Pteridophyta et Spermatophyta) Deutschlands. Schriftenreihe Vegetationsk 28:21-187

Krahulec F, Marhold K, Schmid B (1999) Ecology of closely related plant species: an introduction. Folia Geobot 34:1-5

Kruckeberg AR, Rabinowitz D (1985) Biological aspects of endemism of higher plants. Annual Rev Ecol Syst 16:447-479

Kunin WE, Gaston J (1993) The biology of rarity: patterns, causes and consequences. Trends Ecol Evol 8:298-301

Maschinski J, Baggs JE, Sacchi CF (2004) Seedling recruitment and survival of an endangered limestone endemic in its natural habitat and experimental reintroduction sites. Amer J Bot 91:689-698

Maunder M (1992) Plant reintroduction - an overview. Biodivers \& Conservation 1:51-61

McCune B, Keon D (2002) Equations for potential annual direct incident radiation and head load. $J$ Veg Sci 13:603-606

Meusel H, Jäger E, Weinert E (1965) Vergleichende Chorologie der zentraleuropäischen Flora, 1. Gustav Fischer, Jena

Münzbergová Z (2005) Determinants of species rarity: Population growth rates of species sharing the same habitat. Amer J Bot 92:1987-1994

Münzbergová Z, Herben T (2004) Identification of suitable unoccupied habitats in metapopulation studies using co-occurrence of species. Oikos 105:408-414

Münzbergová Z, Herben T (2005) Seed, dispersal, microsite, habitat and recruitment limitation: identification of terms and concepts in studies of limitations. Oecologia 145:1-8

Murray BR, Thrall PH, Gill AM, Nicotra AB (2002) How plant life-history and ecological traits relate to species rarity and commonness at varying spatial scales. Austral Ecol 27:291-310

Ozinga WA, Schaminee JHJ, Bekker RM, Bonn S, Poschlod P, Tackenberg O, Bakker J, van Groenendael JM (2005) Predictability of plant species composition from environmental conditions is constrained by dispersal limitation. Oikos 108:555-561

Podlech D (1988) Revision von Astragalus L. sect. Caprini DC. (Leguminosae). Mitt Bot Staatssamml München 25:1-924

Pokorný P (2005) Role of man in the development of Holocene vegetation in Central Bohemia. Preslia $77: 113-128$

Rabinowitz D (1981) Seven forms of rarity. In Synge H (ed) The biological aspects of rare plant conservation. Wiley, Chichester, pp 205-217

Rennwald E (ed) (2000): Verzeichnis und Rote Liste der Pflanzengesellschaften Deutschlands. Schriftenreihe Vegetationsk 35:1-800

Schemske DW, Husband BC, Ruckelshaus MH, Goodwillie C, Parker IM, Bishop JG (1994) Evaluating approaches to the conservation of rare and endangered plants. Ecology 75:584-606

Schnittler M, Günther KF (1999) Central European vascular plants requiring priority conservation measures - an analysis from national Red Lists and distribution maps. Biodivers \& Conservation 8:891-925

Scholz P (2000) Katalog der Flechten und flechtenbewohnenden Pilze Deutschlands. Schriftenreihe Vegetationsk 31:1-298

Sparks TH, Menzel A (2002) Observed changes in seasons: an overview. Int J Climatol 22:1715-1725

SPSS Inc (2005) SPSS 15.0 for Windows and Smart-ViewerTM. SPSS Inc., Chicago

Sternberg M, Brown VK, Masters GJ, Clarke IP (1999) Plant community dynamics in a calcareous grassland under climate change manipulations. Pl Ecol 143:29-37

Svenning J-C (2002) A review of natural vegetation openness in north-western Europe. Biol Conservation 104:133-148

SYSTAT Software Inc (2004) SYSTAT 11. Systat Software Inc., Point Richmond

Tackenberg O, Poschlod P, Bonn S (2003) Assessment of wind dispersal potential in plant species. Ecol Monogr 73:191-205

Tear TH, Scott JM, Hayward PH, Griffith B (1995) Recovery plans and the endangered species act: are criticisms supported by data? Conservation Biol 9:182-195

Ter Braak CJF, Šmilauer P (2002) CANOCO Reference Manual and CanoDraw for Windows User's Guide: Software for Canonical Community Ordination (version 4.5). Microcomputer Power, Ithaca

Thompson J (2005) Plant evolution in the Mediterranean. Oxford University Press, Oxford 
Tremlová K, Münzbergová Z (2007) Importance of species traits for species distribution in fragmented landscapes. Ecology 88:965-977

Voss N (2002) Reproduktionsbiologische Untersuchungen an den Steppenpflanzen Astragalus exscapus L., Oxytropis pilosa (L.) DC. und Scorzonera humilis L. Diploma Thesis, University of Göttingen, Göttingen

Walter H, Straka H (1970) Arealkunde, Floristisch-historische Geobotanik. Ed 2. Eugen Ulmer, Stuttgart Westrich P (1989) Die Wildbienen Baden-Württembergs. Eugen Ulmer, Stuttgart

Wilkinson DM (2001) Is local provenance important in habitat creation? J Appl Ecol 38:1371-1373

Wisskirchen R, Haeupler H (1998) Standardliste der Farn- und Blütenpflanzen Deutschlands. Eugen Ulmer, Stuttgart

Young AS, Chang S-M, Sharitz RR (2007) Reproductive ecology of a federally endangered legume, Baptisia arachnifera, and its more widespread congener, B. lanceolata (Fabaceae). Amer J Bot 94:228-236

Youtie BA, Miller RF (1986) Insect seed predation on Astragalus filipes and A. purshii seeds. Northw Sci $60: 42-46$

Received: 1 June 2009 /Revised: 6 April 2010 /Accepted: 9 April 2010 /

Published online: 25 June 2010 